\title{
Outcome Prediction Using Perfusion Parameters and Collateral Scores of Multi-Phase and Single-Phase CT Angiography in Acute Stroke: Need for One, Two, Three, or Thirty Scans?
}

\author{
Katharina Schregel, ${ }^{\mathrm{a},{ }^{*}}$ Ioannis Tsogkas, ${ }^{\mathrm{a},{ }^{*}}$ Carolin Peter, ${ }^{\mathrm{a}}$ Antonia Zapf, ${ }^{\mathrm{b}, \mathrm{c}}$ Daniel Behme, ${ }^{\mathrm{a}}$ \\ Marlena Schnieder, ${ }^{\mathrm{d}}$ Ilko L. Maier, ${ }^{\mathrm{d}}$ Jan Liman, ${ }^{\mathrm{d}}$ Michael Knauth, ${ }^{\mathrm{a}}$ Marios-Nikos Psychogios ${ }^{\mathrm{a}}$ \\ anstitute of Neuroradiology, University Medical Center Goettingen, Goettingen, Germany \\ bDepartment of Medical Statistics, University Medical Center Goettingen, Goettingen, Germany \\ Institute of Medical Biometry and Epidemiology, University Medical Center Hamburg-Eppendorf, Hamburg, Germany \\ 'Department of Neurology, University Medical Center Goettingen, Goettingen, Germany
}

Background and Purpose Collateral status is an important factor determining outcome in acute ischemic stroke (AIS). Hence, different collateral scoring systems have been introduced. We applied different scoring systems on single- and multi-phase computed tomography (CT) angiography (spCTA and mpCTA) and compared them to CT perfusion (CTP) parameters to identify the best method for collateral evaluation in patients with AIS.

Methods A total of 102 patients with AIS due to large vessel occlusion in the anterior circulation who underwent multimodal CT imaging and who were treated endovascularly were included. Collateral status was assessed on spCTA and mpCTA using four different scoring systems and compared to CTP parameters. Logistic regression was performed for predicting favorable outcome. Results All collateral scores correlated well with each other and with CTP parameters. Comparison of collateral scores stratified by extent of perfusion deficit showed relevant differences between groups $(P<0.01$ for each). An spCTA collateral score discriminated best between favorable and unfavorable outcome as determined using the modified Rankin Scale 3 months after stroke.

Conclusions Collateral status evaluated on spCTA may suffice for outcome prediction and decision making in AIS patients, potentially obviating further imaging modalities like mpCTA or CTP.
Correspondence: Marios-Nikos

Psychogios

Institute of Neuroradiology,

University Medical Center

Goettingen, Robert-Koch-Strasse 40,

Goettingen, Germany

Tel: $+49-5513966661$

Fax: +49-513912868

E-mail: m.psychogios@med.uni-goettingen.de

*These authors contributed equally to the manuscript as first author.

Received: February 26, 2018

Revised: August 17, 2018

Accepted: August 31, 2018

Keywords Acute stroke; Collateral circulation; Perfusion; Computed tomography angiography; Treatment outcome

\section{Introduction}

Clinical outcome in acute ischemic stroke (AIS) due to large vessel occlusion (LVO) is influenced by severity and duration of ischemia as well as volume and eloquence of the brain tissue involved.' Hence, both swift reperfusion achieved with endovascu- lar treatment (EVT) and good collaterals have been shown to positively impact outcome. ${ }^{2,3}$ Collateral status can be assessed using digital subtraction angiography, ${ }_{1}^{4}$ magnetic resonance imaging, ${ }^{5,6}$ computed tomography (CT) angiography (CTA), ${ }^{7-10}$ and CT perfusion (CTP) images. ${ }^{11} \mathrm{~A}$ variety of different scoring systems have been established ${ }^{7-9,12-14}$ and it has been shown that 
collateral status can predict infarct size ${ }_{1}^{15}$ outcome, ${ }_{1}^{16}$ response to recanalization, ${ }^{17}$ and treatment effect. ${ }^{18}$ Moreover, poor collateral status was an exclusion criterion for EVT in the Endovascular Treatment for Small Core and Anterior Circulation Proximal Occlusion with Emphasis on Minimizing CT to Recanalization Times (ESCAPE) trial. ${ }^{19}$ While the importance of collaterals in the setting of AIS is indisputable, it remains controversial, which evaluation and scoring method is suited best in an acute clinical setting and whether collateral assessment is an alternative to established techniques like CTP.

Therefore, we applied four different scoring systems to evaluate collateral status on single-phase CTA (spCTA) and multiphase CTA ( $\mathrm{mpCTA}$ ) and compared the results to CTP parameters. The aim of this study was to identify a collateral scoring system, which provides an alternative to CTP and reliably predicts clinical outcome.

\section{Methods}

\section{In- and exclusion-criteria}

Patients were retrospectively identified from our prospective thrombectomy database according to the following inclusion criteria. (1) Multi-modal CT examination consisting of a nonenhanced CT (NECT), whole-brain CTP, and spCTA of the head. (2) Isolated anterior circulation occlusion. (3) Thrombectomy with newer generation of devices (e.g., large-bore aspiration catheter, stent-retriever). Exclusion criteria were severe motion artifacts and incomplete delineation of the intracranial arteries on either CTP or spCTA.

Our prospective database is approved by the Institutional Ethics Committee and contains clinical, angiographic, and follow-up information of all thrombectomy patients treated in our department. Radiographic data are evaluated by a board-certified neuroradiologist with $>5$-year experience, while clinical data (National Institute of Health Stroke Scale [NIHSS], modified Rankin Scale [mRS], etc.) by a certified stroke neurologist.

\section{Image acquisition}

All images were acquired with a 128-slice multidetector CT scanner (Somatom Definition AS+, Siemens Healthineers, Forchheim, Germany) and included a non-contrast CT of the head, a whole-brain CTP and an SpCTA of the extra- and intracranial-arteries, as described before. ${ }^{11}$ CTP included 30 consecutive spiral scans of the brain $(96 \mathrm{~mm}$ in the $\mathrm{z}$-axis, 2 seconds delay after start of contrast agent injection, 45 seconds total acquisition time, $80 \mathrm{kV}, 200 \mathrm{mAs}$, and effective dose of approximately $5 \mathrm{mSv}$ ) after intravenous injection of $36 \mathrm{~mL}$ contrast agent at a rate of $6 \mathrm{~mL} /$ second followed by $30 \mathrm{~mL}$ of saline chaser at a rate of $6 \mathrm{~mL} / \mathrm{second}$ through a cubital vein. CTA was acquired with a single-phase protocol $(120 \mathrm{kV}, 120$ reference $\mathrm{mAs}, 0.3$ second rotation time, 0.6 pitch, $2 \times 64 \times 0.6 \mathrm{~mm}$ collimation, approximately $3 \mathrm{mSv}$ ) after intravenous injection of 60 $\mathrm{mL}$ of contrast agent at a rate of $6 \mathrm{~mL} /$ second, followed by 30 $\mathrm{mL}$ of saline chaser at $6 \mathrm{~mL} / \mathrm{second}$. Bolus triggering for spCTA was placed at the aortic arch (120 HU threshold; 5 seconds delay for bolus watch, 3 seconds delay after reaching threshold).

\section{Image reconstruction and analysis}

Early ischemic changes were evaluated on NECT using the Alberta Stroke Program Early CT Score (ASPECTS).$^{20}$ Time-resolved three-dimensional perfusion scans (four-dimensional [4D] CTA) and spCTA were processed using commercial software packages (Volume Perfusion CT Neuro and 4D Application, Syngo XWP, Siemens). Perfusion maps were reconstructed with a delay-invariant deconvolution method, an automatic motion correction as well as a dedicated noise reduction technique for dynamic data and analyzed with ASPECTS as described before ${ }^{21}$ by a board-certified neuroradiologist with $>5$-year experience in stroke diagnostics.

Temporal maximum intensity projections (tMIPs) were reconstructed from the fusion of the entire CTP datasets into one CTA image with a slice thickness of $24 \mathrm{~mm}$ and a slice distance of $3 \mathrm{~mm}$. In order to simulate mpCTA protocols, we reconstructed the single scans from the 4D CTA into a group of 2 or 3 maximum intensity projection (MIP) reconstructions with the same thickness/distance described above (24/3). We simulated a three-phase mpCTA ( $3 p-m p C T A)$ protocol, as described by Menon et al., ${ }^{13}$ by choosing the first phase according to the peak arterial phase of the 4D CTA and reconstructing the two following phases with a temporal resolution of 7.5 seconds. In order to simulate a two-phase mpCTA ( $2 p-m p C T A)$ protocol, as described by Psychogios et al., ${ }^{22,23}$ we again chose the first phase according to the peak arterial phase and reconstructed the second phase MIPs with a temporal resolution of $10 \mathrm{sec}-$ onds. MIPs were also generated from the spCTA scans with the same slice thickness/distance (24/3).

To assess the inter-rater agreement, all CTA MIPs were then rated by a senior neuroradiologist (M.K.), resident neuroradiologist (I.T.), and medical student (C.P.) by using the following collateral grades:

(1) tMIPs were rated with the single-phase Menon score (sp Menon score). ${ }^{14}$ The sp Menon score, as described in his latest iteration, is a $2 \times 5$-point score of the anterior-media and media-posterior collaterals ( 0 to 5 points for each territory) with a minimal value of 0 (absent collaterals) and a maximum value of 10 (increased or normal collaterals compared with the as- 


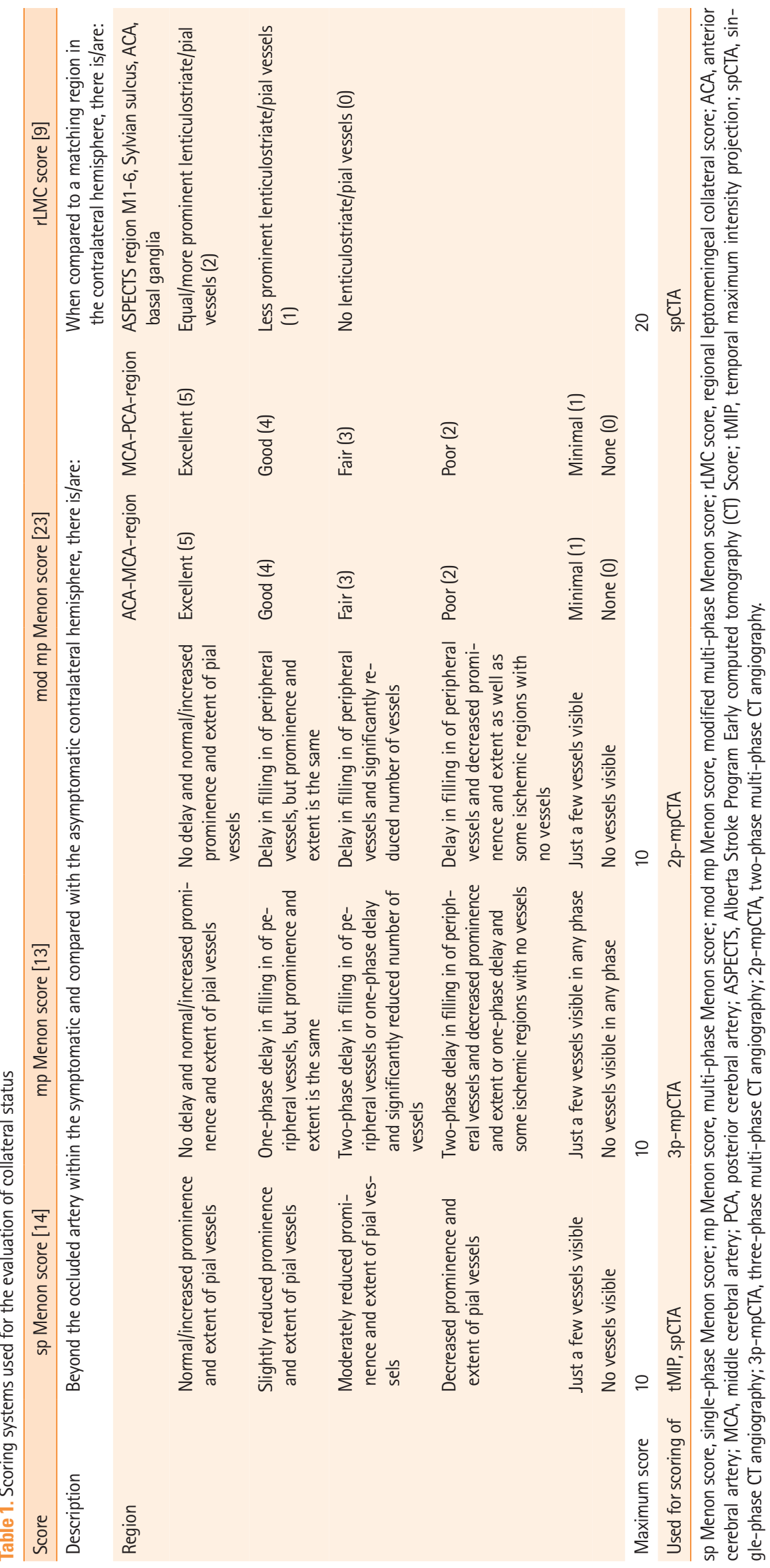



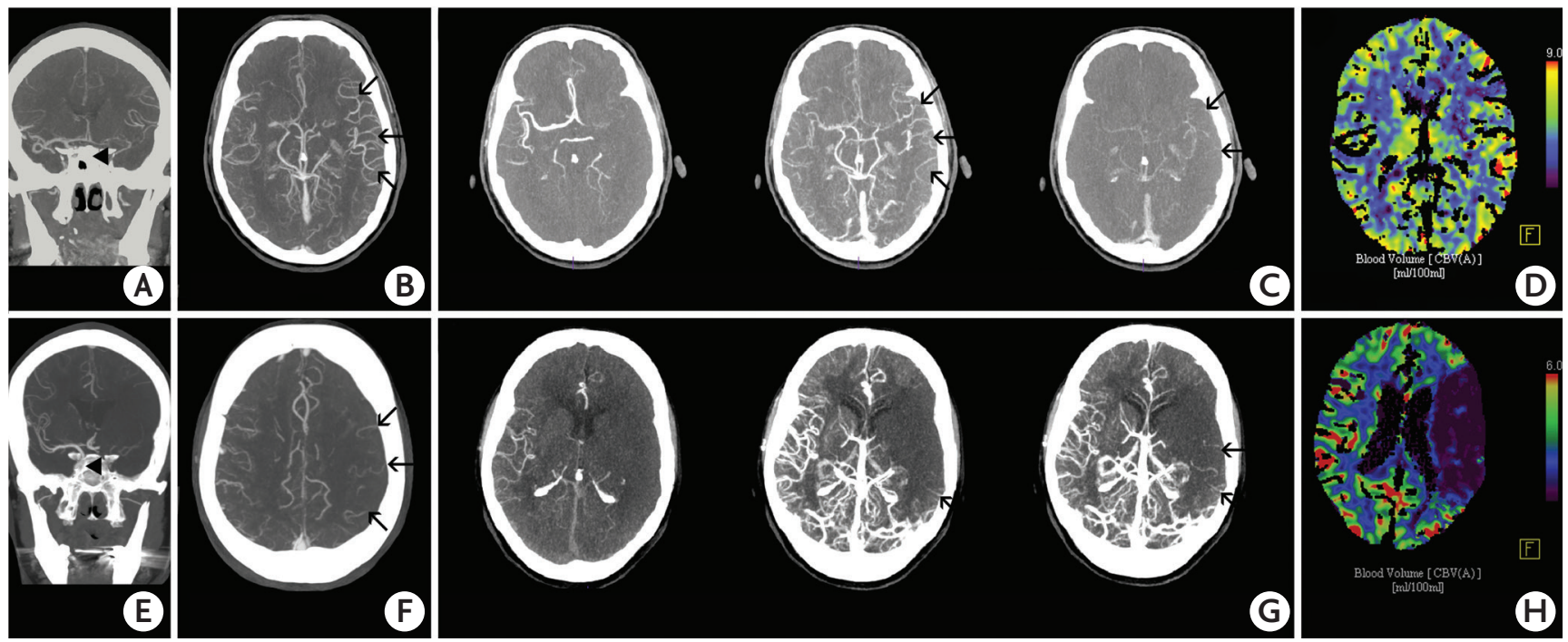

Figure 1. Exemplary images of two patients with an occlusion of the left carotid terminus and excellent (top row, A-D) and poor collateral flow (bottom row, $\mathrm{E}-\mathrm{H}$ ). The occlusion of the distal left internal carotid artery can be identified on coronal reformations of the single-phase computed tomography (CT) angiography (spCTA) (A, E: arrowheads). Collateral flow was evaluated on spCTA images (B, F) using the single-phase Menon score (sp Menon score), ${ }_{1}^{14}$ which compares collaterals within the symptomatic to the contralateral hemisphere. Patient A (top row) had increased prominence and extent of pial vessels within the left symptomatic hemisphere on spCTA images ( $B$, arrows) corresponding to a sp Menon score of 10. In contrast, on the spCTA of patient $B$ (bottom row) just a few pial vessels were visible in the symptomatic hemisphere ( $F$, arrows) resulting in a sp Menon score of 2. Additionally, collateral flow was analyzed on three-phase multi-phase CT angiography (3p-mpCTA) images (,$G)$ applying the multi-phase Menon score (mp Menon score). ${ }^{13}$ The 3p-mpCTA protocol was simulated by choosing the first phase according to the peak arterial phase of the $4 \mathrm{D}$ CTA (first image in $\mathrm{C}, \mathrm{G}$ ) and reconstructing the two following phases with a temporal resolution of 7.5 seconds (second and third images in C, G). Patient A exhibited a one-phase delay in filling in of peripheral vessels within the symptomatic hemisphere (arrows in the second and third image in C), but their prominence and extent was similar to the contralateral hemisphere. This resulted in a mp Menon score of 8. Patient B had a one phase delay in filling in of peripheral vessels (arrows in the second and third image in $\mathrm{G}$ ) and some ischemic regions with no vessels, corresponding to a mp Menon score of 2. Cerebral blood volume (CBV) maps of the two patients are shown in D and H. Patient A had a rather small CBV deficit in the left frontal middle cerebral artery territory and the lentiform nucleus depicted in purple (D). The patient was scored with a CBV-Alberta Stroke Program Early CT Score (ASPECTS) of 7. In contrast, patient B had a CBV deficit in the complete left ACM territory (purple area in H), corresponding to a CBV-ASPECTS of 0.

ymptomatic hemisphere) (Table 1).

(2) 3p-mpCTA MIPs were rated with the multi-phase Menon score ( $m p$ Menon score), ${ }_{1}^{13}$ also with a range of 0 to 10 and distributed points for the pial collaterals of the anterior-medial territory and medial-posterior territory (Table 1).

(3) $2 p$-mpCTA MIPs were rated with the modified multiphase Menon score (mod mp Menon score). ${ }^{23}$ Again, a range of 10 points was used (Table 1).

(4) spCTA MIPs were evaluated with the same sp Menon score used for the tMIPs reconstructions and additionally with the regional leptomeningeal collateral (rLMC) score. ${ }^{9}$ The rLMC grading system (Table 1) is a 20-point scale based on the ASPECTS score, with a range of 0 to 2 points for the opacification of every ASPECTS region of the affected hemisphere $(0$, no pial vessels; 1 , less prominent; 2 , equal/more prominent pial vessel compared to the ASPECTS region of the opposite hemisphere).

All three raters were blinded regarding clinical and follow-up data and evaluated single-phase and multi-phase scores with a 30-day break in between the ratings to reduce recall bias. A senior neuroradiologist prospectively evaluates CTP for every stroke patient in our center and assigns ASPECTS scores to the following parameters: cerebral blood volume (CBV), cerebral blood flow (CBF), time to drain (TTD), and mean transit time (MTT). CBV-ASPECTS and the difference between CBV- and CBF-ASPECTS ( $\triangle \mathrm{CBV}-\mathrm{CBF}$ ) were used for comparative analyses with the collateral scores, as these were previously identified as best predictors for clinical outcome. ${ }^{24}$ Figure 1 shows exemplary images of two patients with an occlusion of the left carotid terminus and excellent collaterals in one case and poor collaterals in the other.

\section{Statistical analysis}

Analyses were performed using MedCalc Statistical Software version 17.6 (MedCalc Software bvba, Ostend, Belgium; http:// www.medcalc.org; 2017) and SAS 9.4 (SAS Institute Inc., Cary, NC, USA). Baseline parameters were analyzed descriptively. As all metric variables followed a skewed distribution, as descriptive measures for them as well as for ordinal variables median and quartiles (interquartile range) were calculated. For binary variables absolute and relative frequencies are provided.

The collateral scores of the senior neuroradiologist are used for all correlations and outcomes analyses. Correlations be- 
tween the different collateral scores as well as compared to the CBV-ASPECTS score were evaluated with the Spearman's rank correlation coefficient. Patients were trichotomized in three groups according to their CBV-ASPECTS grades (0 to 4, 5 to 7 , and 8 to 10) reflecting severity of perfusion deficits. Collateral scores of these groups were compared with the Kruskal-Wallis test and the Mann-Whitney test was used for pairwise comparisons.

Then, patients were dichotomized according to their clinical outcome using mRS after 3 months (90-day mRS) as primary outcome measure. In cases where 90-day mRS was not available, mRS at discharge was used instead. Outcome was considered favorable when 90-day mRS was smaller or equal 2 and unfavorable if 90-day mRS was larger than 2 . The two independent groups with favorable or unfavorable outcome were compared regarding metric and ordinal variables using Mann-Whitney tests. For binary variables the two groups were compared using the chi-square test or Fisher's exact test (for cell frequencies $<5$ ). As sensitivity analysis, we defined favorable outcome more strictly as 90-day mRS 0 to 1 and compared these patients to those with a 90-day $\mathrm{mRS}>1$, which were considered to have an unfavorable outcome. All analyses were repeated using this dichotomization. Because of collinearity between the individual CTP scores and between the individual collateral scores, two multiple logistic regression models with backward selection were used to select independent relevant CTP and one collateral score. Afterwards, a multiple regression model including these selected scores and all other clinical and demographic variables, which demonstrated differences in the univariate analysis $(P<0.2)$. Using backward selection, the final model was fitted. Selected variables were further examined by receiver operating characteristic (ROC) analysis regarding a favorable outcome as defined as a 90-day mRS smaller or equal 2. Sensitivity, specificity as well as positive and negative predictive values were calculated together with the two-sided 95\% confidence intervals (Cls) for the cutoff-values defined by the Youden index. Inter-rater agreement statistic was evaluated with Fleiss' $\mathrm{k}$. Because of multiple comparisons and corresponding multiplicity issues, all $P$-values are used in a descriptive manner, without claiming significance.

\section{Results}

A total of 102 patients (54 female) met the inclusion criteria. The median patient age was 76 years and they were admitted to the hospital with a median NIHSS of 16 and median mRS of 5 , respectively. Most patients had a cardioembolic source of stroke (52\%), while only $7 \%$ of cases were due to large artery atherosclerosis. Another $12 \%$ of patients had embolic strokes of undetermined source, while 5\% of cases were due to other reasons. The etiology was unknown in $24 \%$ of patients. The source of stroke was not relevantly different between patients with a favorable and those with an unfavorable outcome. A favorable outcome was observed in 40/102 patients (39\%) at follow-up. A comparison of baseline characteristics of patients with favorable outcome and those with unfavorable outcome showed that patients with a favorable outcome were younger (66 years vs. 78 years, $P<0.01$ ), had a shorter time from symptom onset to admission (75 minutes vs. 102 minutes, $P=0.1$ ), were less hypertensive ( $60 \%$ vs. $85.5 \%$ of patients, $P \leq 0.01$ ), had less frequent peripheral artery disease (2.6\% vs. $11.5 \%$, $P=0.14$ ) and presented with milder symptoms (median NIHSS admission 14.5 vs. 18 and median mRS admission 4 vs. 5 ; $P=0.02$ and $P=0.01$, respectively) (Table 2). Additionally, the percentage of middle cerebral artery (MCA) occlusions was higher in patients with a favorable outcome (87.5\% vs. $67.7 \%$, $P=0.03$ ) (Table 2). They also had less early ischemic changes on initial NECT (NECT-ASPECTS 9 vs. 8, $P<0.01$ ). When comparing CTP-ASPECT scores, patients with a favorable outcome had higher median CBV-, TTD-, and MTT-ASPECTS than those with an unfavorable outcome ( 8 vs. $7,2.5$ vs. 1 , and 3 vs. $1.5 ; P<0.01$ for each) (Table 2). Additionally, patients with a favorable outcome were assigned higher values in the rLMC score as well as the sp Menon score for both spCTA and tMIP (14 vs. 12,8 vs. 6 , and 9 vs. $7 ; P \leq 0.01$ for each) (Table 2).

The results in patient characteristics remained nearly the same after defining a favorable clinical outcome as 90-day $\mathrm{mRS} 0$ to 1 and an unfavorable outcome as 90-day mRS 2 to 6 (Table 3). As the differences in the results were negligible, further analyses used the initial dichotomization.

Positive correlations were observed between all collateral scores as well as with the CBV-ASPECTS score (Spearman's $r$ between 0.56 and 0.78 ). Correlations were highest between the sp Menon score evaluated on SpCTA and the rLMC score $(r=0.83 ; 95 \% \mathrm{Cl}, 0.76$ to 0.88$)$ and between the sp Menon score evaluated on tMIPs and the mp Menon score $(r=0.73$; $95 \% \mathrm{Cl}, 0.62$ to 0.81$)$. When comparing the collateral scores stratified across the three CBV-ASPECTS groups, relevant differences between the groups could be found for all scores $(P<0.01$ for each score for overall as well as for pairwise comparisons) (Figure 2).

Backward selection lead to the sp Menon score evaluated on spCTA as the collateral score with the strongest association to the outcome (odds ratio [OR], $1.55 ; 95 \% \mathrm{Cl}, 1.17$ to 2.05 ), and to the CBV-ASPECTS as CTP score with the strongest impact (OR, $1.73 ; 95 \% \mathrm{Cl}, 1.27$ to 2.35 ). The overall final model revealed that the probability of a favorable outcome decreases with increasing 
Table 2. Patient characteristics stratified by outcome

\begin{tabular}{|c|c|c|c|}
\hline Characteristic & $\begin{array}{l}\text { Patients with favorable outcome } \\
\text { (90-day } m R S \leq 2)(n=40)\end{array}$ & $\begin{array}{l}\text { Patients with unfavorable outcome } \\
(90 \text {-day } m R S>2)(n=62)\end{array}$ & $P$ \\
\hline Age (yr) & $66(58-75)$ & $78(72-81)$ & $<0.01$ \\
\hline Time from symptom onset to admission (min) & $75(48-118)$ & $102(64-183)$ & 0.1 \\
\hline \multicolumn{4}{|l|}{ Comorbidities } \\
\hline Hyperlipidemia & $19(47.5)$ & $24(39.3)$ & 0.42 \\
\hline Hypertension & $24(60)$ & $53(85.5)$ & $<0.01$ \\
\hline Diabetes & $8(20.5)$ & $16(26.2)$ & 0.51 \\
\hline PAD & $1(2.6)$ & $7(11.5)$ & 0.14 \\
\hline \multicolumn{4}{|l|}{ Stroke etiology } \\
\hline Large artery atherosclerosis & $3(7.5)$ & $4(6.7)$ & 1 \\
\hline Cardioembolic & $21(52.5)$ & $31(51.7)$ & 1 \\
\hline ESUS & $5(12.5)$ & $7(11.7)$ & 1 \\
\hline Other & $3(7.5)$ & $2(3.3)$ & 0.39 \\
\hline Unknown & $8(20)$ & $16(26.7)$ & 0.48 \\
\hline \multicolumn{4}{|l|}{ Clinical scores } \\
\hline NIHSS admission & $14.5(9-18)$ & $18(12-20)$ & 0.02 \\
\hline mRS admission & $4(3.5-5)$ & $5(4-5)$ & 0.01 \\
\hline \multicolumn{4}{|l|}{ Occlusion sites } \\
\hline Proximal ICA & $0(0)$ & $1(1.6)$ & 1 \\
\hline Distal ICA & $5(12.5)$ & $19(30.6)$ & 0.05 \\
\hline MCA & 35 (87.5) & $42(67.7)$ & 0.03 \\
\hline \multicolumn{4}{|l|}{ Imaging scores } \\
\hline NECT-ASPECTS & $9(8-9)$ & $8(7-9)$ & $<0.01$ \\
\hline \multicolumn{4}{|l|}{ CTP } \\
\hline CBV-ASPECTS & $8(7-9)$ & 7 (5-8) & $<0.01$ \\
\hline CBF-ASPECTS & $4(2-5)$ & $3(1-5)$ & 0.06 \\
\hline TTD-ASPECTS & $2.5(1-4)$ & $1(0-3)$ & $<0.01$ \\
\hline MTT-ASPECTS & $3(2-4)$ & $1.5(0-3)$ & $<0.01$ \\
\hline$\triangle \mathrm{CBV}-\mathrm{CBF}-\mathrm{ASPECTS}$ & $4(2.5-5)$ & $3(1-4)$ & 0.04 \\
\hline$\triangle \mathrm{CBV}-\mathrm{TTD}-\mathrm{ASPECTS}$ & $5(4-6)$ & $4(3-6)$ & 0.11 \\
\hline$\triangle \mathrm{CBV}-\mathrm{MTT}$-ASPECTS & $5(4-6)$ & $4(3-6)$ & 0.18 \\
\hline \multicolumn{4}{|l|}{ Collaterals } \\
\hline rLMC score & $14(12-16)$ & $12(10-14)$ & $<0.01$ \\
\hline sp Menon score (CTA) & $8(6-9)$ & $6(4-8)$ & $<0.01$ \\
\hline sp Menon score (tMIP) & $9(7-9)$ & $7(6-8)$ & 0.01 \\
\hline mp Menon score & $7(6-8)$ & $7(5-8)$ & 0.05 \\
\hline mod mp Menon score & $7(6-8)$ & $7(6-8)$ & 0.13 \\
\hline
\end{tabular}

Values are presented as median (interquartile range) or number (\%).

$m R S$, modified Rankin Scale; PAD, peripheral artery disease; ESUS, embolic stroke of undetermined source; NIHSS, National Institute of Health Stroke Scale; ICA, internal carotid artery; MCA, middle cerebral artery; NECT, non-enhanced computed tomography (CT); ASPECTS, Alberta Stroke Program Early CT Score; CTP, CT perfusion; CBV, cerebral blood volume; CBF, cerebral blood flow; TTD, time to drain; MTT, mean transit time; rLMC score, regional leptomeningeal collateral score; sp Menon score, single-phase Menon score; CTA, CT angiography; tMIP, temporal maximal intensity projection; mp Menon score, multi-phase Menon score; mod mp Menon score; modified multi-phase Menon score. 
Table 3. Patient characteristics stratified by outcome

\begin{tabular}{|c|c|c|c|}
\hline Characteristic & $\begin{array}{l}\text { Patients with favorable outcome } \\
\text { (90-day } m R S \leq 1)(n=33)\end{array}$ & $\begin{array}{l}\text { Patients with unfavorable outcome } \\
(90 \text {-day } m R S \geq 2)(n=69)\end{array}$ & $P$ \\
\hline Age (yr) & $67(58-76)$ & $77(68-81)$ & $<0.01$ \\
\hline Time from symptom onset to admission (min) & $75(51-107)$ & $98(60-177)$ & 0.20 \\
\hline \multicolumn{4}{|l|}{ Comorbidities } \\
\hline Hyperlipidemia & $17(51.5)$ & $26(38.2)$ & 0.28 \\
\hline Hypertension & $20(60.6)$ & $57(82.6)$ & 0.03 \\
\hline Diabetes & $7(21.9)$ & $17(25)$ & 0.81 \\
\hline PAD & $1(3)$ & $7(10.4)$ & 0.27 \\
\hline \multicolumn{4}{|l|}{ Stroke etiology } \\
\hline Large artery atherosclerosis & $2(6.1)$ & $5(7.5)$ & 1 \\
\hline Cardioembolic & $18(54.5)$ & $34(50.7)$ & 0.83 \\
\hline ESUS & $4(12.1)$ & $8(11.9)$ & 1 \\
\hline Other & $3(9.1)$ & $2(3)$ & 0.33 \\
\hline Unknown & $6(18.2)$ & $18(26.9)$ & 0.46 \\
\hline \multicolumn{4}{|l|}{ Clinical scores } \\
\hline NIHSS admission & $13(8.8-17.3)$ & $18(12-20)$ & 0.03 \\
\hline mRS admission & $4(3-5)$ & $5(4-5)$ & 0.01 \\
\hline \multicolumn{4}{|l|}{ Occlusion sites } \\
\hline Proximal ICA & $0(0)$ & $1(1.4)$ & 1 \\
\hline Distal ICA & $3(9.1)$ & $21(30.4)$ & 0.03 \\
\hline MCA & 30 (90.9) & $47(68.1)$ & 0.01 \\
\hline \multicolumn{4}{|l|}{ Imaging scores } \\
\hline NECT-ASPECTS & $9(8-9.3)$ & $8(7-9)$ & $<0.01$ \\
\hline \multicolumn{4}{|l|}{ СTP } \\
\hline CBV-ASPECTS & $8(7-9)$ & $7(5-8)$ & $<0.01$ \\
\hline CBF-ASPECTS & $4(2.8-5)$ & $3(1-5)$ & 0.05 \\
\hline TTD-ASPECTS & $3(1-4)$ & $1(0-3)$ & $<0.01$ \\
\hline MTT-ASPECTS & $3(2-5)$ & $2(0.8-3)$ & $<0.01$ \\
\hline$\triangle \mathrm{CBV}$-CBF-ASPECTS & $3(2.8-5.3)$ & $3(1.8-4)$ & 0.08 \\
\hline$\triangle \mathrm{CBV}$-TTD-ASPECTS & $5(4-6)$ & $4(3-6)$ & 0.17 \\
\hline$\triangle \mathrm{CBV}-\mathrm{MTT}$-ASPECTS & $5(4-6)$ & $4(3-6)$ & 0.19 \\
\hline \multicolumn{4}{|l|}{ Collaterals } \\
\hline rLMC score & $15(12-16)$ & $12.5(10-14)$ & $<0.01$ \\
\hline sp Menon score (CTA) & $8(6-9)$ & $6(4.8-8)$ & $<0.01$ \\
\hline sp Menon score (tMIP) & $8.5(7-9)$ & $7(6-8)$ & $<0.01$ \\
\hline mp Menon score & $7(6-8)$ & 7 (5-8) & 0.03 \\
\hline mod mp Menon score & $7.5(6-8)$ & $7(6-8)$ & 0.06 \\
\hline
\end{tabular}

Values are presented as median (interquartile range) or number (\%). A 90-day $m R S \leq 1$ was defined as favorable outcome, while a 90 -day $m R S>1$ was considered unfavorable.

mRS, modified Rankin Scale; PAD, peripheral artery disease; ESUS, embolic stroke of undetermined source; NIHSS, National Institute of Health Stroke Scale; ICA, internal carotid artery; MCA, middle cerebral artery; NECT, non-enhanced computed tomography (CT); ASPECTS, Alberta Stroke Program Early CT Score; CTP, CT perfusion; CBV, cerebral blood volume; CBF, cerebral blood flow; TTD, time to drain; MTT, mean transit time; rLMC score, regional leptomeningeal collateral score; sp Menon score, single-phase Menon score; CTA, CT angiography; tMIP, temporal maximal intensity projection; mp Menon score, multi-phase Menon score; mod mp Menon score; modified multi-phase Menon score. 


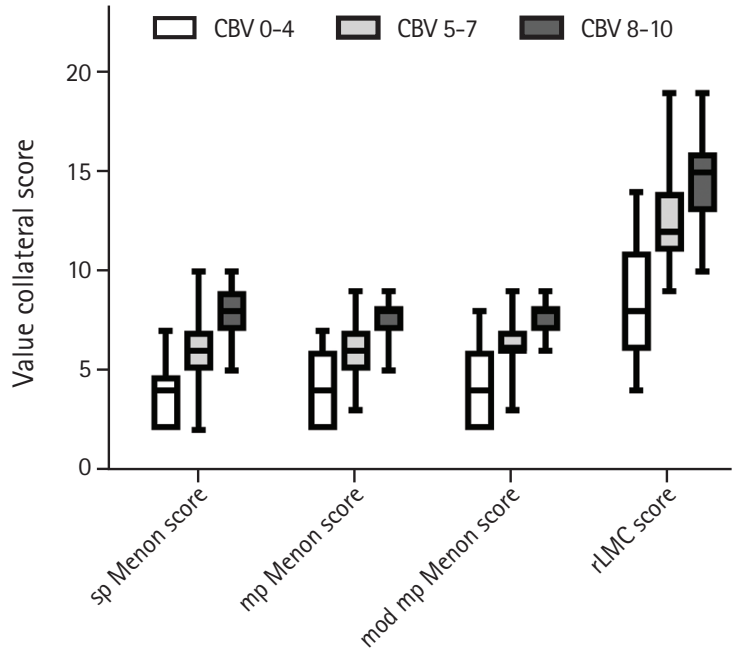

Figure 2. Boxplots of collateral scores stratified by cerebral blood volume (CBV) groups are shown. Patients were trichotomized in three groups according to their CBV-Alberta Stroke Program Early CT Score (ASPECTS) grades (0 to 4, shown in white; 5 to 7, shown in light grey; and 8 to 10 , shown in dark grey) reflecting severity of perfusion deficits and the collateral scores were compared between these groups. A $P<0.01$ for all comparisons. sp Menon score, single-phase Menon score; mp Menon score, multi-phase Menon score; mod mp Menon score, modified multi-phase Menon score; rLMC score, regional leptomeningeal collateral score.

age $(\mathrm{OR}, 0.93 ; 95 \% \mathrm{Cl}, 0.89$ to 0.97$)$, and increases with increasing CBV-ASPECTS (OR, 1.74; 95\% Cl, 1.21 to 2.49 ). Additionally, the probability of a favorable outcome decreases, if patients have an occlusion of the distal internal carotid artery (ICA) compared to an occlusion of the M1- or M2-segment of the MCA $(\mathrm{OR}, 0.19 ; 95 \% \mathrm{Cl}, 0.05$ to 0.72$)$. As only one patient in our cohort had an occluded proximal ICA, the comparison of this occlusion site to the MCA was not meaningful.

ROC analysis including the four collateral scores, CBV-ASPECTS and the difference between $\triangle \mathrm{CBV}$-CBF is demonstrated in Table 4. The analysis showed that the sp Menon score, CBVASPECTS, and the rLMC score discriminated best between favorable and unfavorable outcome (area under the curve [AUC] $0.72,95 \% \mathrm{Cl}, 0.62$ to 0.81 ; AUC $0.71,95 \% \mathrm{Cl}, 0.61$ to 0.80 ; and AUC $0.70,95 \% \mathrm{Cl}, 0.60$ to 0.79 ) (Table 4 and Figure 3). For a value $>5$ in the $s p$ Menon score, sensitivity was $92.5 \%$ and specificity $41.9 \%$. With a favorable outcome prevalence of $39 \%$, the positive predictive value of $\mathrm{sp}$ Menon score $>5$ was $50.5 \%$ and the negative predictive value $89.7 \%$. For CBV-ASPECTS $>7$, the analysis yielded a sensitivity of $62.5 \%$, a specificity of $69.4 \%$, and positive and negative predictive values of $56.6 \%$ and $74.3 \%$, respectively. For rLMC score $>13$, sensitivity was $64.1 \%$ and specificity was $28.8 \%$. The positive predictive value of rLMC score $>13$ was $53.5 \%$ with a negative predictive value of $73.7 \%$. In our analysis, the sp Menon score performed

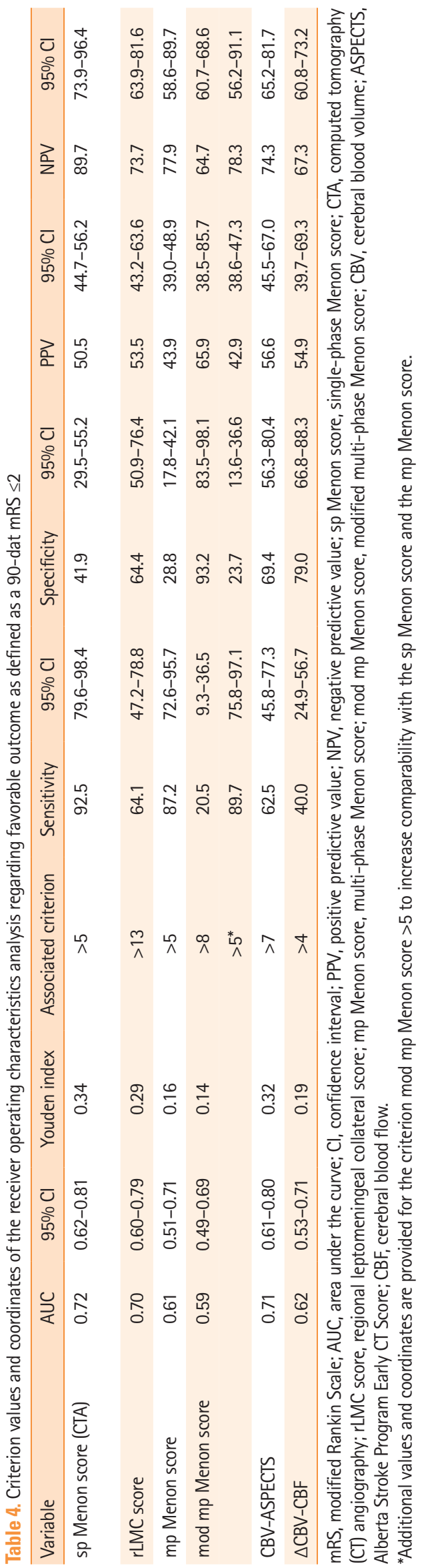




\section{ROC analysis}

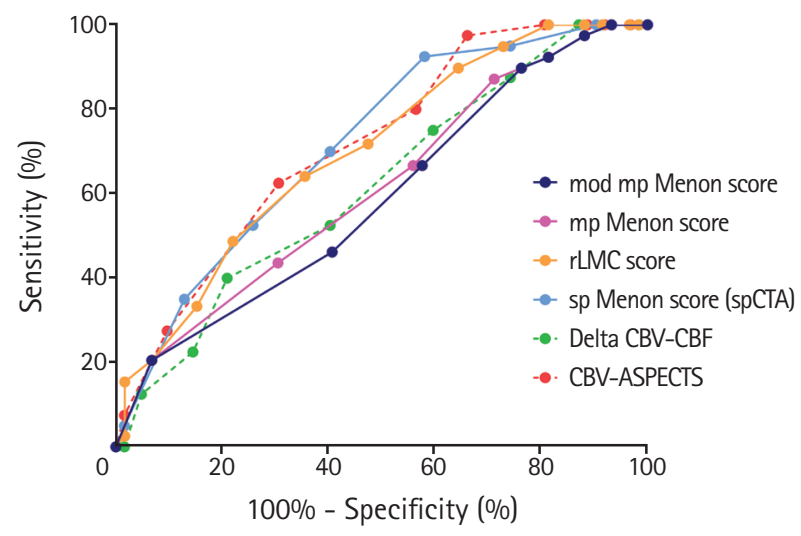

Figure 3. The comparison of receiver operating characteristic (ROC) analyses regarding favorable outcome as defined as a 90-day modified Rankin Scale $\leq 2$ of collateral scores and computed tomography (CT) perfusion parameters is shown. The single-phase Menon score (sp Menon score; blue, solid line), cerebral blood volume (CBV)-Alberta Stroke Program Early CT Score (ASPECTS; red, dashed line), and the regional leptomeningeal collateral score (rLMC score; orange, solid line) discriminate best between favorable and unfavorable outcome. The scores assessed on single-phase CT angiography (spCTA; sp Menon and rLMC score) perform relevantly better than those derived from multi-phase CT angiography (multi-phase Menon score [mp Menon score), pink, solid line; and modified multi-phase Menon score [mod mp Menon score], violet, solid line). CBF, cerebral blood flow.

relevantly better than the $\mathrm{mp}$ Menon score and the mod $\mathrm{mp}$ Menon score (difference between AUCs 0.11 and 0.13, respectively; $P=0.03$ and $P=0.01$, respectively). The performance of the sp Menon and the rLMC score was not relevantly different (difference between AUCs 0.02, $P=0.55$ ).

Inter-rater agreement was very good between the senior neuroradiologist and the resident for all collateral scores ( $\mathrm{k}$ rLMC score $0.96,95 \% \mathrm{Cl}, 0.94$ to 0.98 ; $\mathrm{k}$ sp Menon score 0.89, 95\% Cl, 0.84 to $0.94 ; \mathrm{K} \mathrm{mp}$ Menon score $0.94,95 \% \mathrm{Cl}, 0.91$ to 0.97; and $\mathrm{k}$ mod $\mathrm{mp}$ Menon score $0.90,95 \% \mathrm{Cl}, 0.86$ to 0.95 ). The inter-rater agreement between the medical student and the senior neuroradiologist and the resident, respectively, was only moderate.

\section{Discussion}

This analysis indicates that information on collateral status derived from spCTA by applying the sp Menon score may be sufficient to predict clinical outcome of patients with AIS and could be used for clinical decision making. Our findings suggest that both sp Menon and rLMC scores discriminate equally well between favorable and unfavorable outcome as CBV-ASPECTS and that they performed relevantly better than simulated $\mathrm{mp}$ and $\bmod \mathrm{mp}$ Menon scores. The sp Menon score had the high- est sensitivity to predict clinical outcome. The rLMC score and CBV-ASPECTS were comparable in this regard. Inter-rater agreement between the senior and the resident neuroradiologist was very good for all collateral scores used. The sensitivity analysis with a stricter definition of favorable outcome yielded negligible differences in the results.

All collateral scores correlated well with CBV-ASPECTS. As collaterals and CTP parameters are associated and reflect similar pathophysiologic aspects of AIS, our findings may further corroborate the hypothesis that collateral assessment could supersede perfusion imaging. ${ }^{25,26}$ Even though our final model revealed that the probability of a favorable outcome decreases with increasing age, a proximal occlusion site and decreasing CBV-ASPECTS, we hypothesize that collateral evaluation on spCTA using the sp Menon score may suffice in an acute setting. This score had the strongest association to the outcome. As spCTA is routinely used in the setting of AIS to confirm or exclude $\mathrm{LVO}^{27}$ anyhow, the images are readily available for evaluation of collateral status. By using collateral status as an alternative to CTP parameters for clinical decision making and outcome prediction, the time required for CTP acquisition, reconstruction and evaluation could be saved, reducing total time from hospital admission to treatment. Another advantage is that a similar collateral scoring approach can be applied to flat-panel detector CTA, which is acquired along with a flat-panel detector CT in AIS patients that are managed with a one stop approach. ${ }^{23}$

Menon et al. ${ }^{13}$ implemented a collateral score applicable on mpCTA and showed that this score discriminated clinical outcome of patients with AIS slightly better than CTP or collateral status derived from spCTA images. However, the spCTA images used in this study were acquired in an early arterial phase, while the spCTA we assessed captured the capillary phase. Hence, a direct comparison between our and the spCTA used by Menon et al. ${ }^{13}$ is difficult. Another study assessed additional mpCTA parameters such as washout of contrast agent in pial vessels as well as extent and delay of pial vessel enhancement in the ASPECTS regions and compared these to CTP. ${ }^{26}$ The authors found that the washout on mpCTA predicted follow-up infarction with similar accuracy as CTP and hypothesized that regional collateral assessment on mpCTA may be sufficient for clinical decision making. ${ }^{26}$ In our analysis, the mpCTA collateral scores did not perform better in outcome prediction than the scores derived from spCTA. Hence, there might be no need for other imaging modalities than NECT and spCTA in the acute clinical setting as the important clinical decision for or against EVT can be made based on information gained from NECT and spCTA. Intracranial hemorrhage can be excluded using NECT and presence or absence of LVO as well as collateral status can 
be determined on spCTA. This is not only advantageous due to the saving of time when performing and analyzing spCTA instead of additional mpCTA or CTP, but also with regard to radiation dose. The mean estimated effective radiation dose of our spCTA is $3 \mathrm{mSv}$, while the doses for CTP and mpCTA are 3.5 to $5 \mathrm{mSv}^{13,21}$ and $6 \mathrm{mSv}_{1}^{13}$ respectively. This is especially important given that more patients undergo EVT.

Timing of spCTA is important for correct collateral evaluation, because collateral flow can be underestimated in early arterial phases or overestimated in late venous phases, respectively. ${ }^{11,13}$ However, this did not seem to have been problematic in our analysis as the sp Menon score assessed on spCTA performed better in outcome prediction than the sp Menon score assessed on tMIPs, which are sensitive for late-filling pial vessels while avoiding wash-out phenomena.." The reason for this is likely due to our SpCTA protocol, where $60 \mathrm{~mL}$ contrast agent were administered and a threshold of $120 \mathrm{HU}$ was used for bolus triggering in the aortic arch. The spCTA images acquired according to this protocol are better suited to visualize collaterals than spCTA images derived from a protocol, which used $45 \mathrm{~mL}$ contrast agent and a bolus triggering threshold of $100 \mathrm{HU}_{1}{ }^{21}$ where the collateral status could not be assessed as well.

Limitations of our study include those inherent with a small sample size and a retrospective design of the study, even though prospectively acquired data were used. Hence, the results might be biased by ascertainment and referral patterns. Collateral assessment in general can be confounded by poor cardiac output or flow limiting proximal stenosis. Additionally, the current scoring systems exclusively rate collaterals in the anterior circulation and cannot be used in patients with posterior circulation stroke as the posterior collateral hemodynamics are not as well understood yet. Furthermore, $2 p-$ and $3 p-m p C$ TA images were derived from CTP.

\section{Conclusions}

In summary, collateral status evaluated on spCTA using the sp Menon score may be sufficient for outcome prediction and decision making in the setting of AIS, potentially obviating further imaging modalities like mpCTA or CTP. Further studies to confirm our findings and to test the clinical utility of collateral scoring on spCTA are warranted.

\section{Disclosure}

The Institute of Diagnostic and Interventional Neuroradiology. University Medical Center Goettingen has a research agreement with Siemens Healthcare. Marios-Nikos Psychogios, Daniel Beh- me, and Michael Knauth have received travel grants from Siemens Healthcare. Marios-Nikos Psychogios and Daniel Behme are consultants for Phenox and have received travel grants and minor honorary from Stryker, Acandis, and Penumbra.

\section{References}

1. Sheth $S A$, Liebeskind DS. Collaterals in endovascular therapy for stroke. Curr Opin Neurol 2015;28:10-15.

2. Elijovich L, Goyal N, Mainali S, Hoit D, Arthur AS, Whitehead $M$, et al. CTA collateral score predicts infarct volume and clinical outcome after endovascular therapy for acute ischemic stroke: a retrospective chart review. J Neurointerv Surg 2016;8:559-562.

3. Gersing AS, Schwaiger BJ, Kleine JF, Kaesmacher J, Wunderlich S, Friedrich B, et al. Clinical outcome predicted by collaterals depends on technical success of mechanical thrombectomy in middle cerebral artery occlusion. J Stroke Cerebrovasc Dis 2017;26:801-808.

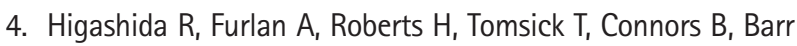
$J$, et al. Trial design and reporting standards for intraarterial cerebral thrombolysis for acute ischemic stroke. J Vasc Inter Radiol 2003;14:S493-S494.

5. Ruland S, Ahmed A, Thomas K, Zhao M, Amin-Hanjani S, Du $X$, et al. Leptomeningeal collateral volume flow assessed by quantitative magnetic resonance angiography in large-vessel cerebrovascular disease. J Neuroimaging 2009;19:27-30.

6. McVerry F, Liebeskind DS, Muir KW. Systematic review of methods for assessing leptomeningeal collateral flow. AJNR Am J Neuroradiol 2012;33:576-582.

7. Tan JC, Dillon WP, Liu S, Adler F, Smith WS, Wintermark M. Systematic comparison of perfusion-CT and CT-angiography in acute stroke patients. Ann Neurol 2007;61:533-543.

8. Maas MB, Lev MH, Ay H, Singhal AB, Greer DM, Smith WS, et al. Collateral vessels on $\mathrm{CT}$ angiography predict outcome in acute ischemic stroke. Stroke 2009;40:3001-3005.

9. Menon BK, Smith EE, Modi J, Patel SK, Bhatia R, Watson TW, et al. Regional leptomeningeal score on CT angiography predicts clinical and imaging outcomes in patients with acute anterior circulation occlusions. AJNR Am J Neuroradiol 2011;32:1640-1645.

10. Sallustio F, Motta C, Pizzuto S, Diomedi M, Giordano A, D'Agostino VC, et al. CT angiography-based collateral flow and time to reperfusion are strong predictors of outcome in endovascular treatment of patients with stroke. $J$ Neurointerv Surg 2017;9:940-943.

11. Frölich AM, Wolff SL, Psychogios MN, Klotz E, Schramm R, Wasser $K_{1}$ et al. Time-resolved assessment of collateral flow 
using 4D CT angiography in large-vessel occlusion stroke. Eur Radio/ 2014;24:390-396.

12. Tan BY, Wan-Yee $K$, Paliwal $P$, Gopinathan $A$, Nadarajah $M$,

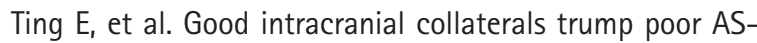
PECTS (Alberta Stroke Program Early CT Score) for intravenous thrombolysis in anterior circulation acute ischemic stroke. Stroke 2016;47:2292-2298.

13. Menon BK, d'Esterre CD, Qazi EM, Almekhlafi M, Hahn L, Demchuk AM, et al. Multiphase CT angiography: a new tool for the imaging triage of patients with acute ischemic stroke. Radiology 2015;275:510-520.

14. Menon BK, Qazi E, Nambiar V, Foster LD, Yeatts SD, Liebeskind $D$, et al. Differential effect of baseline computed tomographic angiography collaterals on clinical outcome in patients enrolled in the interventional management of stroke III trial. Stroke 2015;46:1239-1244.

15. Souza LC, Yoo AJ, Chaudhry ZA, Payabvash S, Kemmling A, Schaefer PW, et al. Malignant CTA collateral profile is highly specific for large admission DWI infarct core and poor outcome in acute stroke. AJNR Am J Neuroradiol 2012;33:1331-1336.

16. Lima FO, Furie $K L$, Silva GS, Lev $M H$, Camargo $E C$, Singhal $A B$, et al. The pattern of leptomeningeal collaterals on CT angiography is a strong predictor of long-term functional outcome in stroke patients with large vessel intracranial occlusion. Stroke 2010;41:2316-2322.

17. Nambiar V, Sohn SI, Almekhlafi MA, Chang HW, Mishra S, Qazi $E_{1}$ et al. CTA collateral status and response to recanalization in patients with acute ischemic stroke. AJNR Am J Neuroradiol 2014;35:884-890.

18. Berkhemer OA, Jansen IG, Beumer D, Fransen PS, van den Berg LA, Yoo AJ, et al. Collateral status on baseline computed tomographic angiography and intra-arterial treatment effect in patients with proximal anterior circulation stroke. Stroke 2016;47:768-776.

19. Goyal M, Demchuk AM, Menon BK, Eesa M, Rempel JL, Thornton J, et al. Randomized assessment of rapid endovascular treatment of ischemic stroke. N Engl J Med 2015;372:1019-1030.
20. Barber PA, Demchuk AM, Zhang J, Buchan AM. Validity and reliability of a quantitative computed tomography score in predicting outcome of hyperacute stroke before thrombolytic therapy. ASPECTS Study Group. Alberta Stroke Programme Early CT Score. Lancet 2000;355:1670-1674.

21. Tsogkas I, Knauth M, Schregel K, Behme D, Wasser K, Maier I, et al. Added value of CT perfusion compared to CT angiography in predicting clinical outcomes of stroke patients treated with mechanical thrombectomy. Eur Radiol 2016;26:42134219.

22. Psychogios MN, Bähr M, Liman J, Knauth M. One stop management in acute stroke: first mothership patient transported directly to the angiography suite. Clin Neuroradiol 2017;27:389-391.

23. Psychogios MN, Behme D, Schregel K, Tsogkas I, Maier IL, Leyhe $J R$, et al. One-stop management of acute stroke patients: minimizing door-to-reperfusion times. Stroke 2017;48:31523155.

24. Psychogios MN, Schramm P, Frölich AM, Kallenberg K, Wasser $K_{1}$ Reinhardt $L$, et al. Alberta Stroke Program Early CT Scale evaluation of multimodal computed tomography in predicting clinical outcomes of stroke patients treated with aspiration thrombectomy. Stroke 2013;44:2188-2193.

25. Vagal A, Menon BK, Foster LD, Livorine A, Yeatts SD, Qazi $E_{1}$ et al. Association between CT angiogram collaterals and CT perfusion in the interventional management of stroke III Trial. Stroke 2016;47:535-538.

26. d'Esterre CD, Trivedi A, Pordeli P, Boesen M, Patil S, Ahn SH, et al. Regional comparison of multiphase computed tomographic angiography and computed tomographic perfusion for prediction of tissue fate in ischemic stroke. Stroke 2017:48:939-945

27. McTaggart RA, Ansari SA, Goyal M, Abruzzo TA, Albani B, Arthur $\mathrm{A}$, et al. Initial hospital management of patients with emergent large vessel occlusion (ELVO): report of the standards and guidelines committee of the Society of Neurolnterventional Surgery. J Neurointerv Surg 2017;9:316-323. 